\title{
GROWTH CONDITIONS AND SIMILARITY ORBITS ${ }^{1}$
}

\author{
P. GHATAGE
}

\begin{abstract}
It is proved that an operator $T$ which acts on a separable Hilbert space and whose resolvent satisfies a first-order growth condition inside and outside the unit circle, can be approximated in norm by operators which are similar to a single unitary operator. In particular the 'Markus-operator' can be approximated by similarities of a diagonal unitary operator and is a compact perturbation of the same.
\end{abstract}

The purpose of this note is to study operators whose resolvents satisfy a first-order growth condition inside and outside the unit circle. A. S. Markus has shown by an example that such operators need not be similar to unitary operators. We prove that such an operator always belongs to the normclosure of the similarity orbit of a unitary operator.

We denote the similarity orbit of $T$ by $\delta(T)=\left\{S T S^{-1}, S\right.$ bounded and invertible $\}$. The spectrum, essential spectrum and left essential spectrum are denoted by $\sigma(T), \sigma_{e}(T)$ and $\sigma_{e l}(T)$ respectively, and the image of $T$ in the Calkin algebra is denoted by $\pi(T)$.

Throughout this paper we assume that $\mathcal{H}$ is a separable Hilbert space.

Our main tool is a recent theorem due to Herrero, which to the best of our knowledge is still unpublished. Hence we state it here for reference.

THEOREM A. Let $N$ be a normal operator acting on a separable Hilbert space $\mathcal{H}$ and let $T \in L(\mathcal{H})$. In order that $T$ belong to $\mathrm{cl} \delta(N)$ the following conditions are sufficient: (a) $T$ is bi-quasitriangular. (b) $\sigma(T) \supseteq \sigma(N)$ and $\sigma_{e}(T) \supseteq \sigma_{e}(N)$. (c) If $\lambda \in \sigma(T) \backslash \sigma_{e}(T)$ then $\lambda$ is isolated. (d) If $\lambda$ is an isolated point of $\sigma(T)$, then $\mathcal{H}=\mathcal{K}_{1} \oplus \mathcal{K}_{2}=\mathcal{K}_{1}^{1}+\mathcal{H}_{2}^{1}$, where $\mathcal{K}_{1}=\operatorname{ker}(\lambda-N)$ and $\mathcal{H}_{1}^{1}=\operatorname{ker}(\lambda-T)$ have the same dimension, $\mathcal{H}_{2}$ reduces $N$ and has the same dimension as $\mathcal{H}_{2}^{1}, T \mathcal{H}_{2}^{1} \subseteq \mathcal{F}_{2}^{1}$ and $\lambda \notin \sigma\left(T \mid \mathcal{H}_{2}^{1}\right) \cup \sigma\left(N \mid \mathcal{F}_{2}\right)$. (e) If $\lambda \in \sigma_{e}(T)$ is not isolated then the component of $\lambda$ in $\sigma_{e}(T)$ contains some nonisolated point of $\sigma_{e}(N)$. (f) If $\lambda \in \sigma_{e}(T)$ is isolated and $f$ is the analytic function defined by $f(z)=\lambda-z$ in a neighborhood of $\lambda$ and $f(z) \equiv 0$ in an open neighborhood of $\sigma_{e}(T) \mid\{\lambda\}$ then $f[\pi(T)]=0$.

COROLlaRy A. If $N$ is normal and $\sigma(N)$ is perfect then $T \in \mathrm{cl} \delta(N)$ for any biquasitriangular $T$ such that $\sigma(T)=\sigma(N) \sigma_{e}(T)$.

Received by the editors March 11, 1976 and, in revised form, November 16, 1976.

AMS (MOS) subject classifications (1970). Primary 47A99.

Key words and phrases. Quasitriangular, essential spectrum, similarity orbits.

'Work supported partially by the National Research Council of Canada under Grant A-7169. 
We start with the following simple lemma which is a slight modification of a lemma due to Markus, Nikol'skaja and Nikol'skiī (see [5]).

LEMma 1. Suppose that $\sigma(T) \subseteq\{\lambda,|\lambda|=1\}=\Gamma$, and $T$ satisfies the condition, $\left\|(T-\lambda)^{-1}\right\| \leqslant M(|\lambda|-1)^{-1}$ for $|\lambda|>1$. Then for $\lambda_{0} \in \Gamma$ we have,

$$
\operatorname{ker}\left(T-\lambda_{0}\right) \dot{+} \operatorname{cl}\left[\operatorname{Im}\left(T-\lambda_{0}\right)\right]=\mathcal{H} \text {. }
$$

COROLlARY 1. Under the hypotheses of Lemma 1,T is biquasitriangular.

Proof. It follows from Lemma 1 that whenever $\lambda_{0} \in \Gamma, \operatorname{ker}\left(T-\lambda_{0}\right)$ and $\operatorname{ker}\left(T^{*}-\bar{\lambda}_{0}\right)$ have the same dimension. Since $\sigma_{e}(T) \subseteq \sigma(T) \subseteq \Gamma$ it follows from the well-known theorem of Apostol, Foiaş, and Voiculescu [1, p. 13] that $T$ and $T^{*}$ are quasitriangular.

We feel that this corollary may be true without the assumption regarding the growth-condition.

LEMMA 2. Under the hypothesis of Lemma 1, $\sigma_{e}(T)=\{$ limit points of $\sigma(T)\} \cup\{$ isolated eigenvalues of infinite multiplicity $\}$.

Proof. By Putnam's theorem (see [7]) $\partial \sigma(T) \subseteq \sigma_{e l}(T) \cup$ isolated eigenvalues of finite multiplicity $\}$. Since $\sigma(T)=\partial \sigma(T)$ it follows that limit points of $\sigma(T)$ belong to $\sigma_{e}(T)$. Conversely if $\lambda \in \sigma_{e}(T)$ and $\lambda$ is an isolated point of $\sigma(T)$ then by Stampfli's result [9], $\lambda$ is an isolated eigenvalue of $\sigma(T)$ and $\operatorname{ker}(\lambda-T)=$ range of the corresponding Riesz projection. Thus $\mathscr{H}=\operatorname{ker}(\lambda$ $-T)+\mathcal{H}_{0}$ where $\lambda \notin \sigma\left(T \mid \mathcal{F}_{0}\right)$. Since $\lambda \in \sigma_{e}(T)=\sigma_{e l}(T)$, it follows that $\operatorname{ker}(\lambda-T)$ is infinite-dimensional.

LEMma 3. Suppose $\mathfrak{U}$ is a $C^{*}$-algebra with identity and $a \in \mathfrak{A}$ satisfies the properties

(i) $\sigma(a)$ is contained in a $C_{1}$-Jordan curve $\Gamma$,

(ii) $\left\|R_{\lambda}(a)\right\| \leqslant k / \operatorname{dist}(\lambda, \Gamma)$ for $\lambda$ inside $\Gamma$,

(iii) $\left\|R_{\lambda}(a)\right\| \leqslant k /\{\operatorname{dist}(\lambda, \Gamma)\}^{n}$ for $\lambda$ outside $\Gamma$.

Suppose $\mu$ is an isolated point of $\sigma(a)$ and $p$ is the corresponding Riesz projection. Then $p(a-\mu)=0$.

This is a slightly more general statement of a result due to Stampfli. The proof is no different from that of Lemma 4 of [9].

Proposition 1. If $\sigma(T) \subseteq \Gamma,\left\|(T-\lambda)^{-1}\right\| \leqslant M /|1-| \lambda \|$ whenever $\lambda \notin \Gamma$ and $\bigvee\{\operatorname{ker}(T-\mu), \mu \in \Gamma\}=\mathcal{H}$, then $T \in \mathrm{cl} \mathcal{S}(U)$ for a diagonal unitary operator $U$.

Proof. Note that as a trivial consequence of Lemma 1, for $\lambda \in \Gamma$ therè exists a projection $P_{\lambda}$ on the $\operatorname{ker}(T-\lambda)$ parallel to $\operatorname{Im}(T-\lambda)$ and it can be seen that $\left\|P_{\lambda}\right\| \leqslant M$. Thus for $x$ in $\mathcal{H}$ and any $\varepsilon>0,\left\{\lambda,\left\|P_{\lambda} x\right\|>\varepsilon\right\}$ is finite. Since $\mathcal{H}$ is separable, it follows that there can be only countably many distinct eigenvalues. See [6, p. 1124]. Moreover it follows as in the proof of Theorem 3 of [5] that $\sigma(T)$ is the closure of the set of these eigenvalues. Let $U$ be the diagonal unitary operator defined by repeating each eigenvalue of $T$ as 
often as its multiplicity. Obviously $\sigma(T)=\sigma(U)$. It follows from Weyl's characterization of $\sigma_{e}(U)$ (see [8, p. 367]) and Lemma 2 that $\sigma_{e}(U)=\sigma_{e}(T)$. If $\mu$ is an isolated point of $\sigma(T)$ then it follows from Lemma 3 that $\mu$ is an eigenvalue of $T$ and the eigenspace is equal to the range of the corresponding Riesz projection. Thus condition (d) of Theorem A is satisfied. To see that ( $f$ ) is satisfied we need only note that in the Calkin algebra $\pi(T)=a$ satisfies the same resolvent-growth conditions as $T$ does in $\mathcal{L}(\mathcal{H})$, and apply Lemma 3 to $\pi(T)$. Thus by Theorem A, $T \in \operatorname{cl} \delta(U)$.

Lemma 4. Suppose $\sigma(T) \subseteq \Gamma$ and $\left\|(T-\lambda)^{-1}\right\| \leqslant M /|1-| \lambda||$ for $\lambda \notin \Gamma$. Let $\mathfrak{R}=\bigvee\{\operatorname{ker}(T-\mu), \mu \in \Gamma\}$ and $T_{0}=T \mid \mathfrak{T}$. If $T_{1}=\left.P^{\perp} T\right|^{\mathfrak{N}}$ where $P$ is the orthogonal projection onto $\Re$, then $\sigma\left(T_{1}\right)$ is a perfect subset of $\Gamma$.

Proof. As explained in the proof of Proposition 1, it follows that $\sigma\left(T_{0}\right) \subseteq$ closure of the eigenvalues of $T$. Thus $\sigma\left(T_{0}\right) \subseteq \sigma(T)$ and hence $\sigma\left(T_{1}\right) \subseteq \sigma(T)$.

Since $\left\|\left(T^{*}-\bar{\lambda}\right)^{-1}\right\| \leqslant M /|1-| \lambda||$ it follows that $T_{1}^{*}-\bar{\lambda}$ (being a restriction of $\left.T^{*}-\bar{\lambda}\right)$ is bounded below by $|1-| \lambda|| / M$. This, together with the fact $\sigma\left(T_{1}\right) \subseteq \sigma(T) \subseteq \Gamma$, proves that the resolvent of $T_{1}$ satisifes the same conditions as the resolvent of $T$. Hence by Lemma 3 any isolated point of $\sigma\left(T_{1}\right)$ is an eigenvalue of $T_{1}$. Thus it is sufficient to prove that $T_{1}$ has no eigenvalues on $\Gamma$. Suppose the contrary. Without loss of generality suppose that 1 is an eigenvalue of $T_{1}$, and consider the restriction of $T$ to

$$
\mathfrak{N}^{1}=\Re \vee \operatorname{ker}\left(T_{1}-I\right) \text {. }
$$

We call it $S$. Applying Lemma 1 to $S$ we have $\operatorname{ker}(S-I)+\operatorname{Im}(S-I)$ $=\mathfrak{R}^{1}$. Obviously $\operatorname{Im}(S-I) \subseteq \Re$, and since $S$ is a restriction of $T$, $\operatorname{ker}(S-I) \subseteq \operatorname{ker}(T-I)$. Now it follows from the definition of $\mathfrak{N}$ that $\mathfrak{N}^{1} \subseteq \mathfrak{R}$. This is clearly a contradiction.

Theorem 1. Suppose $T$ is as in the previous proposition. Then there exists a unitary operator $U$ and a sequence $\left\{S_{n}\right\}$ of invertible operators such that whenever $f$ is analytic in a neighborhood of the unit circle, $\| f(T)-$ $S_{n} f(U) S_{n}^{-1} \| \rightarrow 0$. Moreover $U$ can be chosen such that $\sigma(T)=\sigma(U)$ and $\sigma_{e}(T)=\sigma_{e}(U)$. If in addition $\sigma_{e}(T)$ is finite then there exists a compact operator $K$ and an invertible operator $S$ such that $T=S U S^{-1}+K$.

Proof. (i) We first prove the existence of a unitary operator $U$ such that $T \in \mathrm{cl} \delta(U)$. To this end define $\Re, P, T_{0}$ and $T_{1}$ as in Proposition 2. Let $U_{0}$ be the diagonal unitary operator on $\mathscr{N}$, defined as in Proposition 1 . Recall that $\sigma\left(T_{0}\right)=\sigma\left(U_{0}\right), \quad \sigma_{e}\left(T_{0}\right)=\sigma_{e}\left(U_{0}\right), \quad \sigma\left(T_{0}\right) \cup \sigma\left(T_{1}\right)=\sigma(T)$, and by Putnam's theorem [7] $\sigma_{e}\left(T_{1}\right)=\sigma\left(T_{1}\right)$. Since $\sigma_{e}\left(T_{0}\right)=\sigma_{e l}\left(T_{0}\right)$ it follows easily that $\sigma_{e}(T)=\sigma_{e}\left(T_{0}\right) \cup \sigma_{e}\left(T_{1}\right)$. Let $U_{1}$ be any fixed unitary operator on $\Re_{1}$ such that $\sigma\left(U_{1}\right)=\sigma\left(T_{1}\right)$. Since $\sigma\left(U_{1}\right)$ is perfect and $T_{1}$ is biquasitriangular, it follows from Corollary A that $T_{1} \in \operatorname{cl} \delta\left(U_{1}\right)$. Let $U=U_{0} \oplus U_{1}$. Then $U$ is a unitary operator such that

$$
\sigma(U)=\sigma\left(U_{0}\right) \cup \sigma\left(U_{1}\right)=\sigma\left(T_{0}\right) \cup \sigma\left(T_{1}\right)=\sigma(T) \text { and } \sigma_{e}(U)=\sigma_{e}(T) \text {. }
$$


Since any isolated point of $\sigma(T)$ belongs to $\sigma\left(T_{0}\right)$ but not to $\sigma\left(T_{1}\right)$, condition (d) is satisfied by the construction in Proposition 1. A similar reasoning applied to $\sigma_{e}(T)$ shows that (f) is true. Thus by Theorem A, $T \in \operatorname{cl} \delta(U)$.

(ii) Let $\left\{S_{n}\right\}$ be a sequence of invertible operators such that $\| T-$ $S_{n} U S_{n}^{-1} \|<1 / M n$. If $|\lambda|<1-1 / n$, then a simple calculation shows that $\left\|S_{n}(U-\lambda)^{-1} S_{n}^{-1}\right\|<M /(1-|\lambda|-1 / n)$. Similarly if $|\lambda|>1+1 / n$,

$$
\left\|S_{n}(U-\lambda)^{-1} S_{n}^{-1}\right\|<M /(1+1 / n-|<|) .
$$

Thus it is easy to see that whenever $\lambda \notin \Gamma$,

$$
S_{n}(U-\lambda)^{-1} S_{n}^{-1} \rightarrow(T-\lambda)^{-1}
$$

in the norm and the convergence is uniform off any neighborhood of the unit circle. By using the usual Riesz-Dunford functional calculus it can be seen that $\left\|f(T)-S_{n} f(U) S_{n}^{-1}\right\| \rightarrow 0$ whenever $f$ is analytic in an open neighborhood of $\Gamma$.

(iii) If $\sigma_{e}(T)$ is finite then so is $\sigma_{e}(U)$, and it follows from [2, Theorem 3.1] that there exists a compact operator $K$ and an invertible operator $S$ such that $T=S U S^{-1}+K$.

REMARK. If $T$ is the Cayley transform of the operator defined by Markus [4] then $T$ is not similar to any unitary operator. But if $U$ is the corresponding diagonal unitary operator then $\sigma_{e}(U)=\{1\}$, and by Proposition $1, T$ belongs to $\mathrm{cl} \delta(U)$. Since $U-I$ is compact it follows that $T-I$ is compact, and hence $T$ is a compact perturbation of $U$. Thus Markus's example is a particular case of Proposition 1, in which the eigenvectors of $T$ actually form a (conditional) basis for $H$. A constructive proof of Proposition 1 for this special case would be very interesting.

\section{BIBLIOGRAPHY}

1. R. G. Douglas and C. M. Pearcy, Invariant subspaces of non-quasitriangular operators, Proc. Conf. Operator Theory (Dalhousie Univ., Halifax, N. S., 1973), Lecture Notes in Math., vol. 345, Springer, Berlin, 1973, pp. 13-57. MR 50 \# 10857.

2. L. A. Fialkow, The similartiy orbit of a normal operator, Trans. Amer. Math. Soc. 210 (1975), 129-137. MR 51 \# 11152.

3. D. A. Herrero, Closure of similarity orbits of Hilbert space operators. II (to appear).

4. A. S. Markus, Certain criteria for the completeness of a system of root vectors of a linear operator in a Banach space, Mat. Sb. 70 (112) (1966), 526-561; English transl., Amer. Math. Soc. Transl. (2) 85 (1969), 51-91. MR 35 \# 7151.

5. A. S. Markus, L. N. Nikol'skaja and N. K. Nikol'skii, The unitary spectrum of a contraction in a Banach space, Zap. Naucn. Sem. Leningrad Otdel. Mat. Inst. Steklov. (LOMI) 22 (1971), 65-74 = J. Soviet Math. 2 (1974), 112-119. MR 44 \#7337.

6. N. K. Nikol'skin, On spectral analysis on the unitary spectrum. Point spectrum, Dokl. Akad. Nauk SSSR 199 (1971), 544-547 = Soviet Math. Dokl. 12 (1971), 1123-1126. MR 44 \#5805.

7. C. R. Putnam, The spectra of operators having resolvents of first-order growth, Trans. Amer. Math. Soc. 133 (1968), 505-510. MR 37 \#4651.

8. F. Riesz and B. Sz-Nagy, Lecons d'analyse fonctionelle, Akad. Kiadö, Budapest, 1952; English transl., Functional analysis, Ungar, New York, 1955. MR 14, 286; 17, 175.

9. J. G. Stampfli, A local spectral theory for operators. III: Resolvents, spectral sets and similarity, Trans. Amer. Math. Soc. 168 (1972), 133-151. MR 45 \#4182.

Department of Mathematics, University of Toronto, Toronto, Ontario M5S 1A1, CANADA 